\section{Schwierige Krankheitsbilder}

\section{Merkelzell-Karzinom}

\section{Andres}

Klinik und Poliklinik für Dermatologie und Allergologie am Biederstein, Klinikum rechts der Isar, Technische Universität München,

Deutschland

Das Merkelzell-Karzinom (MCC), häufig auch als neuroendokrines Karzinom bezeichnet, ist ein hochmaligner kutaner Tumor. Klinisch präsentiert sich das MCC in der Mehrzahl der Fälle als rötlicher, halbkugelig erhabener, zumeist asymptomatischer Knoten auf Sonnenlicht-exponierter Haut bei überwiegend älterer und/oder immunsupprimierter kaukasischer Bevölkerung. Aufgrund der relativ unspezifischen Klinik des MCC ist für die exakte Diagnostik eine histologische und immunhistochemische Untersuchung unabdingbar. Die Inzidenz des MCC beträgt derzeit circa 0,6/100.000/Jahr und hat sich in den letzten 20 Jahren vervierfacht. Die Prognose des MCC ist schlecht, häufig liegt zum Zeitpunkt der Diagnostik bereits ein metastasiertes Stadium vor. Die aktuell publizierten Fünfjahresüberlebensraten betragen bei Patienten im Stadium der Fernmetastasierung knapp 20\%. Therapeutisch wird neben der radikalen Operation mit ausreichendem Sicherheitsabstand (idealerweise $3 \mathrm{~cm}$ ) die Wächterlymphknoten-Dissektion mit anschließender Radiatio empfohlen. Das Ansprechen von Chemotherapeutika ist derzeit unbefriedigend.

Im Januar 2008 wurde erstmalig das Merkelzell-Polyomavirus $(\mathrm{MCPyV})$ beschrieben, dem eine kausale Rolle in der Tumorigenese des MCC zugeschrieben wird. MCPyV-DNS lässt sich in der überwiegenden Mehrheit im MCC-Gewebe nachweisen (60-90\%). Inwieweit dieser ätiopathogenetische Zusammenhang auch therapeutisch von Nutzen sein wird, werden künftige Studien zeigen.

\section{Neue Therapien}

\section{Immuntherapie bei Hautkrebs}

R. Hein, A. Onken, J. Ring

Klinik und Poliklinik für Dermatologie und Allergologie am Biederstein, Klinikum rechts der Isar, Technische Universität München, Deutschland

Die Inzidenz und Mortalität des malignen Melanoms der Haut ist in den letzten Jahren trotz verbesserter Früherkennungsmaßnahmen und Heilung nach Exzision dünner Melanome deutlich gestiegen. Aktuell nimmt man an, dass die Anzahl der Neuerkrankungen in Deutschland bei 20/100.000 Einwohner pro Jahr liegt. Die Mortalität steigt jedes Jahr um circa $3 \%$ an.

Anfang 2010 wurde vom American Joint Committee on Cancer (AJCC) eine neue Tumorklassifikation für das maligne Melanom herausgegeben. Diese berücksichtigt bei der T-Klassifikation neben der Tumordicke zusätzlich die Mitoserate und die Ulzeration. Leider existiert bislang keine adjuvante Therapie, die das Auftreten von regionären oder Fernmetastasen verhindern können. Interferon- $\alpha$ ist zwar im Stadium II zugelassen, allerdings kommt es nur bei einer kleinen Subgruppe von Patienten mit Mikrometastasierung im Lymphknoten zu einer Verbesserung des Gesamtüberlebens.

In Tumorzellen kommt es durch Genmutationen oder Tanslokationen zu einer Überaktivität von Signalkaskaden, welche beispielsweise die Angiogenese und Proliferation einer Zelle steuern. In den letzten Jahren konnten glücklicherweise auf molekularer Ebene neue Substanzklassen entwickelt werden, die gezielt bei entsprechendem Mutationsnachweis eingesetzt wurden. Hierzu zählen Tyrosinkinaseinhibitoren z.B. Imatinib (Glivec) und Sorafenib, der BRAF-Inhibitor PLX4032 und der CTLA-4-Antagonist Ipilimumab. Zum Teil konnten diese Präparate in Phase II und III Studien bereits vielversprechende Erfolge erzielen.

\section{Anaphylaxie-Schulung}

K. Brockow ${ }^{1}$, S. Schallmayer ${ }^{2}$, K. Beyer ${ }^{3}$, T. Biedermann ${ }^{4}$, J. Fischer ${ }^{4}$, N. Gebert ${ }^{3}$, U. Gieler ${ }^{2}$, M. Grosber ${ }^{1}$, T. Jakob ${ }^{5}$, L. Klimek ${ }^{6}$, C. Kugler ${ }^{1}$, L. Lange ${ }^{7}$, O. Pfaar $^{6}$, E. Rietschel ${ }^{8}$, F. Rueff ${ }^{9}$, S. Schnadt ${ }^{10}$, R. Szczepanski ${ }^{11}$, M. Worm ${ }^{12}$, J. Ring ${ }^{1}$ für die Arbeitsgemeinschaft Anaphylaxie Training und Edukation (AGATE)*

${ }^{1}$ Klinik und Poliklinik für Dermatologie und Allergologie am Biederstein, Technische Universität München, Deutschland; ${ }^{2}$ Klinik für Psychosomatik und Psychotherapie, Universität Gießen, Deutschland; ${ }^{3}$ Klinik für Pädiatrie mit Schwerpunkt Pneumologie und Immunologie, Charité - Universitätsmedizin, Berlin, Deutschland; ${ }^{4}$ UniversitätsHautklinik Tübingen, Deutschland; ${ }^{5}$ Universitäts-Hautklinik Freiburg, Deutschland; 'Zentrum für Allergologie und Rhinologie Wiesbaden der HNO-Universitätsklinik Mannheim, Deutschland; ${ }^{7}$ SanktMarien-Hospital, Bonn, Deutschland; ${ }^{8}$ Zentrum für Kinderheilkunde der Universitätsklinik Köln, Deutschland; ${ }^{9}$ Klinik und Poliklinik für Dermatologie und Allergologie, Klinikum der Ludwig-MaximiliansUniversität, München, Deutschland; ${ }^{10}$ Deutscher Allergie- und Asthmabund, Mönchengladbach, Deutschland; ${ }^{11}$ Kinderhospital Osnabrück, Deutschland; "2 Allergie-Centrum-Charité, Klinik für Dermatologie, Venerologie und Allergologie, Charité - Universitätsmedizin, Berlin, Deutschland

*Weitere beteiligte Institutionen und Mitglieder der Arbeitsgemeinschaft Anaphylaxie Training und Edukation: Imke Reese, Ernährungstherapeutische Praxis München, Deutschland; Frank Friedrich, Pädiatrische Kinderarztpraxis Aachen, Deutschland; Alexander Dorsch, TrainMed, Haimhausen, Deutschland

Hintergrund: Anaphylaxiepatienten mit erhöhtem Risiko für erneute Reaktionen wird ein Notfallset inklusive AdrenalinAutoinjektor verschrieben. Das Wissen und Verhalten bezüglich Allergenmeidung und Selbsttherapie sind zumeist ungenügend.

Methoden: Die Arbeitsgemeinschaft Anaphylaxie Training und Edukation (AGATE) erstellte ein standardisiertes Schulungsprogramm für Patienten oder Erziehungsberechtigte nach Anaphylaxie. In Gruppenschulungen werden sechs Patienten oder zwölf Erziehungsberechtigte geschult. Eine prospektive, kontrollierte und randomisierte Evaluationsstudie wurde in zwölf deutschen Zentren abgeschlossen. Wissensfragen, Verhaltenstest sowie testpsychologische Instrumente 